PROCEEDINGS OF THE

AMERICAN MATHEMATICAL SOCIETY

Volume 132, Number 1, Pages 147-156

S 0002-9939(03)07075-8

Article electronically published on April 24, 2003

\title{
THE FORM SUM AND THE FRIEDRICHS EXTENSION OF SCHRÖDINGER-TYPE OPERATORS ON RIEMANNIAN MANIFOLDS
}

\author{
OGNJEN MILATOVIC \\ (Communicated by David S. Tartakoff)
}

\begin{abstract}
We consider $H_{V}=\Delta_{M}+V$, where $(M, g)$ is a Riemannian manifold (not necessarily complete), and $\Delta_{M}$ is the scalar Laplacian on $M$. We assume that $V=V_{0}+V_{1}$, where $V_{0} \in L_{\text {loc }}^{2}(M)$ and $-C \leq V_{1} \in L_{\text {loc }}^{1}(M)(C$ is a constant) are real-valued, and $\Delta_{M}+V_{0}$ is semibounded below on $C_{c}^{\infty}(M)$. Let $T_{0}$ be the Friedrichs extension of $\left.\left(\Delta_{M}+V_{0}\right)\right|_{C_{c}^{\infty}(M)}$. We prove that the form sum $T_{0} \tilde{+} V_{1}$ coincides with the self-adjoint operator $T_{F}$ associated to the closure of the restriction to $C_{c}^{\infty}(M) \times C_{c}^{\infty}(M)$ of the sum of two closed quadratic forms of $T_{0}$ and $V_{1}$. This is an extension of a result of Cycon. The proof adopts the scheme of Cycon, but requires the use of a more general version of Kato's inequality for operators on Riemannian manifolds.
\end{abstract}

\section{InTRODUCTION AND THE MAIN RESULT}

Let $(M, g)$ be a Riemannian manifold (i.e. $M$ is a $C^{\infty}$-manifold, $\left(g_{j k}\right)$ is a Riemannian metric on $M), \operatorname{dim} M=n$. We will assume that $M$ is connected. We will also assume that we are given a positive smooth measure $d \mu$, i.e. in any local coordinates $x^{1}, x^{2}, \ldots, x^{n}$ there exists a strictly positive $C^{\infty}$-density $\rho(x)$ such that $d \mu=\rho(x) d x^{1} d x^{2} \ldots d x^{n}$. We do not assume that $(M, g)$ is complete.

We will consider a Schrödinger-type operator of the form

$$
H_{V}=\Delta_{M}+V .
$$

Here $\Delta_{M}:=d^{*} d$, where $d: C^{\infty}(M) \rightarrow \Omega^{1}(M)$, and $V \in L_{\text {loc }}^{1}(M)$ is real-valued.

1.1. Maximal operator. We define the maximal operator $H_{V \text {, max }}$ associated to $H_{V}$ as an operator in $L^{2}(M)$ given by $H_{V, \max } u=H_{V} u$ with domain

$$
\operatorname{Dom}\left(H_{V, \max }\right)=\left\{u \in L^{2}(M): V u \in L_{\mathrm{loc}}^{1}(M), H_{V} u \in L^{2}(M)\right\} .
$$

Here $\Delta_{M} u$ in $H_{V} u=\Delta_{M} u+V u$ is understood in the distributional sense.

We make the following assumptions on $V$.

Assumption A. Assume $V=V_{0}+V_{1}$, where

(i) $V_{0} \in L_{\text {loc }}^{2}(M)$ and $\Delta_{M}+V_{0}$ is semibounded below on $C_{c}^{\infty}(M)$.

(ii) $V_{1} \in L_{\text {loc }}^{1}(M)$ and $V_{1} \geq-C$, where $C>0$ is a constant.

Received by the editors August 20, 2002.

2000 Mathematics Subject Classification. Primary 35P05, 58G25; Secondary 47B25, 81Q10.

(C)2003 American Mathematical Society 
1.2. Quadratic forms. For any self-adjoint operator $T: \operatorname{Dom}(T) \subset L^{2}(M) \rightarrow$ $L^{2}(M)$ such that $T \geq-\alpha$, we will denote by $Q(T)$ the domain of the quadratic form $t$ associated to $T$. By Theorem 2.1 in [3], $t$ is a closed semibounded below form, i.e. $Q(T)$ is a Hilbert space with the inner product

$$
(u, v)_{t}=t(u, v)+(1+\alpha)(u, v)_{L^{2}(M)},
$$

where $t(\cdot, \cdot)$ is the sesquilinear form obtained by polarization of $t$.

1.3. Form sum. By (i) of Assumption A, $\Delta_{M}+V_{0}$ is symmetric and semibounded below on $C_{c}^{\infty}(M)$, so we can associate to it a semibounded below self-adjoint operator $T_{0}$ (Friedrichs extension, cf. Theorem 14.1 in [3]).

We will denote by $T_{0} \tilde{+} V_{1}$ the form sum of $T_{0}$ and $V_{1}$. By Theorem 4.1 in [3], this is the self-adjoint operator associated to the semibounded below closed quadratic form $t_{q}$ given by the sum of two semibounded below closed quadratic forms corresponding to $T_{0}$ and $V_{1}$. By the same theorem, the following is true: $Q\left(T_{0} \tilde{+} V_{1}\right)=Q\left(T_{0}\right) \cap$ $Q\left(V_{1}\right)$. Clearly, $T_{0} \tilde{+} V_{1}$ is a self-adjoint restriction of $H_{V, \max }$.

1.4. Operator $T_{F}$. Denote by $t_{\min }$ the restriction of $t_{q}$ to $C_{c}^{\infty}(M) \times C_{c}^{\infty}(M)$. Denote by $T_{F}$ the self-adjoint operator associated to the closure of $t_{\min }$ in the sense of the norm in $Q\left(T_{0} \tilde{+} V_{1}\right)$. Clearly, $T_{F}$ is a self-adjoint restriction of $H_{V \text {,max }}$.

We will give a sufficient condition for $T_{F}=T_{0} \tilde{+} V_{1}$.

Theorem 1.5. Suppose that Assumption A holds. Then $T_{F}=T_{0} \tilde{+} V_{1}$.

Remark 1.6. Theorem 1.5 was proven by Cycon 2 in the case of the operator $-\Delta+V$ in an open set $M \subset \mathbb{R}^{n}$, where $\Delta$ is the standard Laplacian on $\mathbb{R}^{n}$ with the standard metric. In the case $V_{0}=0$ and $M=\mathbb{R}^{n}$ with standard metric, Theorem 1.5 was proven in Simon [13].

\section{Operators With a POSitive Form CORE}

Definition 2.1. Let $T: C_{c}^{\infty}(M) \subset L^{2}(M) \rightarrow L^{2}(M)$ be a symmetric semibounded below operator. Let $T_{F}$ denote its Friedrichs extension and $Q\left(T_{F}\right)^{+}$the set of a.e. positive elements of $Q\left(T_{F}\right)$. We say that $T_{F}$ has a positive form core if for every $u \in Q\left(T_{F}\right)^{+}$there exists a sequence $u_{k} \in C_{c}^{\infty}(M)^{+}$such that

$$
\left\|u_{k}-u\right\|_{t} \rightarrow 0 \quad \text { as } k \rightarrow \infty,
$$

where $\|\cdot\|_{t}$ is the norm associated to the closure of quadratic form $t(v, w):=(T v, w)$ $\left(v, w \in C_{c}^{\infty}(M)\right)$ via (1.2).

The main result of this section is

Theorem 2.2. Suppose that $\Delta_{M}+V_{0}$ is as in (i) of Assumption A. Let $T_{0}$ be the Friedrichs extension of $\left.\left(\Delta_{M}+V_{0}\right)\right|_{C_{c}^{\infty}(M)}$. Then $T_{0}$ has a positive form core.

Remark 2.3. In the case of the operator $-\Delta+V_{0}$ in an open set $M \subset \mathbb{R}^{n}$, Theorem 2.2 was proven in [2] Th. 1].

We will first prove the following special case of Theorem 2.2

Proposition 2.4. Suppose that $-C \leq V_{0} \in L_{\mathrm{loc}}^{2}(M)$, where $C>0$ is a constant. Let $T_{b}$ be the Friedrichs extension of $\left.\left(\Delta_{M}+V_{0}\right)\right|_{C_{c}^{\infty}(M)}$. Then $T_{b}$ has a positive form core. 
We begin with a few preliminary lemmas.

In what follows $T_{b}$ is as in the hypothesis of Proposition 2.4 and $t_{b}$ is the closed quadratic form associated with $T_{b}$. Without loss of generality, we may and we will assume that $V_{0} \geq 0$ so that $T_{b}$ is a positive self-adjoint operator.

We will denote $W^{1,2}(M):=\left\{u \in L^{2}(M): d u \in L^{2}\left(T^{*} M\right)\right\}$. By $W_{0}^{1,2}(M)$ we will denote the closure of $C_{c}^{\infty}(M)$ in the norm $\|u\|_{W^{1,2}}^{2}:=\|d u\|^{2}+\|u\|^{2}$, where $\|\cdot\|$ is the $L^{2}$ norm. By $Q\left(V_{0}\right)$ we will denote the set $\left\{u \in L^{2}(M): V_{0}^{1 / 2} u \in L^{2}(M)\right\}$. Clearly, $Q\left(V_{0}\right)$ is the closure of $C_{c}^{\infty}(M)$ in the norm

$$
\|u\|_{V_{0}}^{2}:=\left\|V_{0}^{1 / 2} u\right\|^{2}+\|u\|^{2}
$$

where $\|\cdot\|$ is the norm in $L^{2}(M)$.

In the proofs of the following three lemmas, we will use the arguments from the proof of Lemma 1 in [5].

Lemma 2.5. $Q\left(T_{b}\right)=W_{0}^{1,2}(M) \cap Q\left(V_{0}\right)$.

Proof. Denote by $\mathcal{H}_{1}:=W_{0}^{1,2}(M) \cap Q\left(V_{0}\right)$. Consider a sesquilinear form $S: \mathcal{H}_{1} \times$ $\mathcal{H}_{1} \rightarrow \mathbb{C}$ given by

$$
S(u, v):=(d u, d v)+\left(V_{0}^{1 / 2} u, V_{0}^{1 / 2} v\right),
$$

where $(\cdot, \cdot)$ is the inner product in $L^{2}$.

This sesquilinear form is closed, so the pre-Hilbert space $\mathcal{H}_{1}$ is complete in the norm

$$
(u, v)_{t_{b}}:=(d u, d v)+\left(V_{0}^{1 / 2} u, V_{0}^{1 / 2} v\right)+(u, v) .
$$

By definition of $W_{0}^{1,2}(M)$ and $Q\left(V_{0}\right)$, it follows that $\mathcal{H}_{1}$ is the closure of $C_{c}^{\infty}(M)$ in the norm $\|\cdot\|_{t_{b}}$ corresponding to (2.2).

For all $u, v \in C_{c}^{\infty}(M),(u, v)_{t_{b}}=(u, v)+\left(T_{b} u, v\right)$. By Theorem 14.1 in [3], $Q\left(T_{b}\right)$ is the closure of $C_{c}^{\infty}(M)$ in the norm $\|\cdot\|_{t_{b}}$ corresponding to (2.2), so $Q\left(T_{b}\right)=$ $W_{0}^{1,2}(M) \cap Q\left(V_{0}\right)$.

Lemma 2.6. Assume that $u \in C_{c}^{\infty}(M)$. Then there exists a sequence $\phi_{k} \in$ $C_{c}^{\infty}(M)^{+}$such that $\left\|\phi_{k}-|u|\right\|_{t_{b}} \rightarrow 0$ as $k \rightarrow \infty$, where $\|\cdot\|_{t_{b}}$ is the norm corresponding to (2.2).

Proof. Let $u \in C_{c}^{\infty}(M)$. Then $|u| \in W_{\text {comp }}^{1,2}(M)$. Using a partition of unity we may assume that $u$ is supported in a coordinate neighborhood. Let $|u|^{\rho}=J^{\rho}|u|$, where $J^{\rho}$ is the Friedrichs mollifying operator; cf. Sect. 5.11 in [1]. Then $|u|^{\rho} \in C_{c}^{\infty}(M)$. It is well-known that $|u|^{\rho} \rightarrow|u|$ as $\rho \rightarrow 0+$ both in the space $W_{\text {comp }}^{1,2}(M)$ and in the space $L_{\text {comp }}^{2}(M)$. Also, since $|u|$ is continuous compactly supported on $M$ and $V_{0} \in L_{\mathrm{loc}}^{2}(M)$, we have

$$
\int V_{0}\left(|u|^{\rho}\right)^{2} d \mu \rightarrow \int V_{0}|u|^{2} d \mu \quad \text { as } \rho \rightarrow 0+.
$$

Therefore,

$$
\left\||u|^{\rho}-|u|\right\|_{t_{b}} \rightarrow 0 \quad \text { as } \rho \rightarrow 0+,
$$

where $\|\cdot\|_{t_{b}}$ is the norm corresponding to (2.2).

Lemma 2.7. Suppose that $u \in Q\left(T_{b}\right)$. Then $|u| \in Q\left(T_{b}\right)$. 
Proof. Let $u \in Q\left(T_{b}\right)$. By Lemma [2.5, we get $u \in W_{0}^{1,2}(M) \cap Q\left(V_{0}\right)$. Since $u \in W_{0}^{1,2}(M)$, Lemma 7.6 from [4] gives $|u| \in W_{0}^{1,2}(M)$. From $u \in Q\left(V_{0}\right)$, we immediately get $|u| \in Q\left(V_{0}\right)$. Therefore, $|u| \in W_{0}^{1,2}(M) \cap Q\left(V_{0}\right)$, so by Lemma 2.5. we obtain $|u| \in Q\left(T_{b}\right)$.

2.8. Proof of Proposition 2.4. We will follow the proof of Lemma 2 in 2 .

Suppose that $u \in Q\left(T_{b}\right)^{+}$. By Lemma 2.5, there exists a sequence $\phi_{j} \in C_{c}^{\infty}(M)$ such that

$$
\left\|\phi_{j}-u\right\|_{t_{b}} \rightarrow 0 \quad \text { as } j \rightarrow \infty
$$

where $\|\cdot\|_{t_{b}}$ is the norm corresponding to (2.2).

In what follows, we will denote $(\operatorname{sign} w)(x):=\frac{w(x)}{|w(x)|}$ when $w(x) \neq 0$, and 0 otherwise.

We have

$$
\begin{aligned}
\left\|\left|\phi_{j}\right|-u\right\|_{t_{b}}^{2} & =\left\|\left|\phi_{j}\right|-u\right\|^{2}+\left\|d\left|\phi_{j}\right|-d u\right\|^{2}+\left\|V_{0}^{1 / 2}\left(\left|\phi_{j}\right|-u\right)\right\|^{2} \\
\leq & \left\|\phi_{j}-u\right\|^{2}+\left\|d\left|\phi_{j}\right|-d u\right\|^{2}+\left\|V_{0}^{1 / 2}\left(\phi_{j}-u\right)\right\|^{2} \\
& =\left\|\phi_{j}-u\right\|^{2}+\left\|\operatorname{Re}\left(\left(\operatorname{sign} \bar{\phi}_{j}\right) d \phi_{j}\right)-d u\right\|^{2}+\left\|V_{0}^{1 / 2}\left(\phi_{j}-u\right)\right\|^{2},
\end{aligned}
$$

where $\|\cdot\|$ denotes the norm $L^{2}$.

From (2.6) we obtain

$$
\begin{aligned}
\left\|\left|\phi_{j}\right|-u\right\|_{t_{b}}^{2} \leq & \left\|\phi_{j}-u\right\|^{2}+\left[\left\|\left(\operatorname{sign} \bar{\phi}_{j}\right)\left(d \phi_{j}-d u\right)\right\|+\left\|\left(\operatorname{sign} \bar{\phi}_{j}-1\right) d u\right\|\right]^{2} \\
& +\left\|V_{0}^{1 / 2}\left(\phi_{j}-u\right)\right\|^{2} \\
\leq & \left\|\phi_{j}-u\right\|^{2}+\left[\left\|d \phi_{j}-d u\right\|+\left\|\left(\operatorname{sign} \bar{\phi}_{j}-1\right) d u\right\|\right]^{2}+\left\|V_{0}^{1 / 2}\left(\phi_{j}-u\right)\right\|^{2},
\end{aligned}
$$

where $\|\cdot\|$ denotes the norm in $L^{2}$.

By (2.5), the first, second and fourth term on the right-hand side of (2.7) go to 0 as $j \rightarrow \infty$.

It remains to show that

$$
\left\|\left(\operatorname{sign} \bar{\phi}_{j}-1\right) d u\right\| \rightarrow 0 \quad \text { as } j \rightarrow \infty .
$$

Since $\phi_{j} \rightarrow u$ in $L^{2}(M)$, a lemma of Riesz shows that there exists a subsequence $\phi_{j_{k}}$ such that $\phi_{j_{k}} \rightarrow u$ a.e. $d \mu$, as $k \rightarrow \infty$. By Lemma 7.7 from [4, it follows that $d u=0$ almost everywhere on $\{x \in M: u(x)=0\}$. Hence, as $k \rightarrow \infty$, sign $\overline{\phi_{j_{k}}} \rightarrow 1$ a.e. on $M$. Since $d u \in L^{2}\left(T^{*} M\right)$, dominated convergence theorem immediately implies (2.8) (after passing to the chosen subsequence $\phi_{j_{k}}$ ).

This shows that

$$
\left\|\left|\phi_{j_{k}}\right|-u\right\|_{t_{b}} \rightarrow 0 \quad \text { as } k \rightarrow \infty .
$$

By (2.9) and Lemma 2.6, there exists a sequence $\psi_{l}$ in $C_{c}^{\infty}(M)^{+}$such that $\left\|\psi_{l}-u\right\|_{t_{b}} \rightarrow 0$ as $l \rightarrow \infty$. By Definition 2.1 it follows that $T_{b}$ has a positive form core.

In what follows, we will use a version of Kato's inequality. For the proof of this inequality in general setting, cf. Theorem 5.6 in [1].

Theorem 2.9. Let $E$ be a Hermitian vector bundle on $M$, and let $\nabla: C^{\infty}(E) \rightarrow$ $C^{\infty}\left(T^{*} M \otimes E\right)$ be a Hermitian connection on $E$. Let $\nabla^{*}: C^{\infty}\left(T^{*} M \otimes E\right) \rightarrow C^{\infty}(E)$ 
be formal adjoint of $\nabla$ with respect to the usual inner product on $L^{2}(E)$. Assume that $u \in L_{\mathrm{loc}}^{1}(E)$ and $\nabla^{*} \nabla u \in L_{\mathrm{loc}}^{1}(E)$. Then

$$
\Delta_{M}|u| \leq \operatorname{Re}\left\langle\nabla^{*} \nabla u, \operatorname{sign} u\right\rangle
$$

where

$$
\operatorname{sign} u(x)= \begin{cases}\frac{u(x)}{|u(x)|} & \text { if } u(x) \neq 0, \\ 0 & \text { otherwise. }\end{cases}
$$

Definition 2.10. Let $(X, \mu)$ be a measure space. A bounded linear operator $A: L^{2}(X, \mu) \rightarrow L^{2}(X, \mu)$ is said to be positivity preserving if for every $0 \leq u \in$ $L^{2}(X, \mu)$ we have $A u \geq 0$.

We will also use the following abstract theorem due to Simon; cf. Theorem 2.1 in [11.

Theorem 2.11 (Simon [1]). Suppose that $(X, \mu)$ is a measure space. Suppose that $H$ is a positive self-adjoint operator in $L^{2}(X, \mu)$. Then $(H+1)^{-1}$ is positivity preserving if and only if the following two conditions are satisfied:

(i) For every $u \in Q(H)$, we have $|u| \in Q(H)$.

(ii) For every $u \in \operatorname{Dom}(H)$ and $0 \leq v \in Q(H)$, the following is true:

$$
\operatorname{Re}[h(|u|, v)] \leq \operatorname{Re}((\operatorname{sign} u) v, H u),
$$

where $h$ is the quadratic form associated to $H$, and $(\operatorname{sign} u)(x)=\frac{u(x)}{|u|}$ whenever $u(x) \neq 0$, and 0 otherwise.

The following lemma extends Lemma 1 from [5] to the case of Riemannian manifolds.

Lemma 2.12. The operator $\left(T_{b}+1\right)^{-1}$ is positivity preserving.

Proof. Let $t_{b}$ be the quadratic form associated to $T_{b}$. By Theorem 2.11 it suffices to check the following conditions:

(i) For every $u \in Q\left(T_{b}\right)$, we have $|u| \in Q\left(T_{b}\right)$ and

(ii) For every $u \in \operatorname{Dom}\left(T_{b}\right)$ and $0 \leq v \in Q\left(T_{b}\right)$, the following is true:

$$
\operatorname{Re}\left[t_{b}(|u|, v)\right] \leq \operatorname{Re}\left((\operatorname{sign} u) v, T_{b} u\right) .
$$

Condition (i) follows immediately by Lemma 2.7

We now prove that condition (ii) holds. Let $u \in \operatorname{Dom}\left(T_{b}\right)$. Then $\left(\Delta_{M}+V_{0}\right) u$ $\in L^{2}(M)$ and hence $\Delta_{M} u \in L_{\text {loc }}^{1}(M)$.

For $u \in \operatorname{Dom}\left(T_{b}\right)$ and $0 \leq \phi \in C_{c}^{\infty}(M)$ we have

$$
\begin{aligned}
\operatorname{Re}\left[t_{b}(|u|, \phi)\right] & =\operatorname{Re}\left(|u|,\left(\Delta_{M}+V_{0}\right) \phi\right)=\left(|u|, \Delta_{M} \phi\right)+\left(|u|, V_{0} \phi\right) \\
& =\left(\Delta_{M}|u|, \phi\right)+\left(V_{0}|u|, \phi\right) \\
& \leq \operatorname{Re}\left((\operatorname{sign} \bar{u}) \Delta_{M} u, \phi\right)+\left((\operatorname{sign} \bar{u}) V_{0} u, \phi\right) \\
& =\operatorname{Re}\left((\operatorname{sign} \bar{u}) T_{b} u, \phi\right)=\operatorname{Re}\left((\operatorname{sign} u) \phi, T_{b} u\right) .
\end{aligned}
$$

Here we used integration by parts and the special case of Kato inequality (2.10) for $\Delta_{M}$.

Let $0 \leq v \in Q\left(T_{b}\right)$. By Proposition 2.4, there exists a sequence $\phi_{j} \in C_{c}^{\infty}(M)^{+}$ such that $\left\|\phi_{j}-v\right\|_{t_{b}} \rightarrow 0$ as $j \rightarrow \infty$, where $\|\cdot\|_{t_{b}}$ is the norm corresponding to (2.2). 
From (2.11), we obtain

$\operatorname{Re}\left[t_{b}(|u|, v)\right]=\lim _{j \rightarrow \infty} \operatorname{Re}\left[t_{b}\left(|u|, \phi_{j}\right)\right] \leq \lim _{j \rightarrow \infty} \operatorname{Re}\left((\operatorname{sign} u) \phi_{j}, T_{b} u\right)=\operatorname{Re}\left((\operatorname{sign} u) v, T_{b} u\right)$.

This proves condition (ii) and the lemma.

In what follows $T_{0}$ is as in the hypothesis of Theorem 2.2. Without loss of generality we may and we will assume that $T_{0}$ is a positive self-adjoint operator.

We will also use the notation $\mathbb{Z}_{+}:=\{1,2,3, \ldots\}$.

Proposition 2.13. $\left(T_{0}+1\right)^{-1}$ is positivity preserving.

Proof. We will adopt the arguments from the proof of Lemma 2 in [5] to our setting.

For every $k \in \mathbb{Z}_{+}$and $x \in M$, define

$$
Q_{k}(x)= \begin{cases}V_{0}(x) & \text { if } V_{0}(x) \geq-k, \\ -k & \text { if } V_{0}(x)<-k .\end{cases}
$$

Let $T_{k}$ be the Friedrichs extension of $\left(\Delta_{M}+Q_{k}\right) \mid C_{c}^{\infty}(M)$. Then for all $k \in \mathbb{Z}_{+}$ and $u \in C_{c}^{\infty}(M)$, we have

$$
\left(u, T_{k} u\right) \geq\left(u, T_{0} u\right) \geq 0,
$$

where $(\cdot, \cdot)$ is the inner product in $L^{2}(M)$.

From (2.12) it follows that

$$
T_{0} \leq T_{k} \quad \text { for all } k \in \mathbb{Z}_{+},
$$

i.e. $Q\left(T_{k}\right) \subset Q\left(T_{0}\right)$, and for all $u \in Q\left(T_{k}\right), t_{0}(u, u) \leq t_{k}(u, u)$, where $t_{0}$ and $t_{k}$ are the quadratic forms associated to $T_{0}$ and $T_{k}$, respectively.

Furthermore, for all $u \in C_{c}^{\infty}(M)$, the following is true:

$$
\left(u, T_{k} u\right) \rightarrow\left(u, T_{0} u\right) \quad \text { as } k \rightarrow \infty .
$$

Clearly, $C_{c}^{\infty}(M) \subset Q\left(T_{k}\right)$ for all $k \in \mathbb{Z}_{+}$. By definition of Friedrichs extension, it follows that $C_{c}^{\infty}(M)$ is dense in $Q\left(T_{0}\right)$ (in the norm of $Q\left(T_{0}\right)$ ).

This, (2.13) and (2.14) show that the hypotheses of abstract Theorem 7.9 from [3] are satisfied.

Therefore, as $k \rightarrow \infty, T_{k} \rightarrow T_{0}$ in the strong resolvent sense.

By Lemma [2.12, $\left(T_{k}+1\right)^{-1}$ is positivity preserving for all $k \in \mathbb{Z}_{+}$. Therefore, $\left(T_{0}+1\right)^{-1}$ is also positivity preserving.

Corollary 2.14. Assume that $u \in Q\left(T_{0}\right)$. Then $|u| \in Q\left(T_{0}\right)$.

Proof. $T_{0}$ is a positive self-adjoint operator in $L^{2}(M)$. By Proposition 2.13 , the operator $\left(T_{0}+1\right)^{-1}$ is positivity preserving. Now the corollary follows immediately from Theorem 2.11.

2.15. Truncation operators corresponding to $T_{0}$. Let $T_{0}$ be as in the hypothesis of Theorem 2.2.

Define $V_{0}^{+}:=\max \left\{V_{0}, 0\right\}, V_{0}^{-}:=\max \left\{-V_{0}, 0\right\}$, and for each $k \in \mathbb{Z}_{+}$, let $V_{0}^{k}:=$ $\min \left\{k, V_{0}^{-}\right\}$.

Denote by $T_{+}$and $T_{k}$ the Friedrichs extension of $\left.\left(\Delta_{M}+V_{0}^{+}\right)\right|_{C_{c}^{\infty}(M)}$ and $\left.\left(\Delta_{M}+V_{0}^{+}-V_{0}^{k}\right)\right|_{C_{c}^{\infty}(M)}$, respectively.

Let $t_{0}, t_{+}$and $t_{k}\left(k \in \mathbb{Z}_{+}\right)$be the quadratic forms associated to $T_{0}, T_{+}$and $T_{k}$, respectively. 
The following lemma is analogous to Lemma 3 in [2].

Lemma 2.16. With the notations of Section 2.15.

(i) $T_{k} \rightarrow T_{0}$ in the strong resolvent sense as $k \rightarrow \infty$.

(ii) $Q\left(T_{+}\right) \subset Q\left(T_{0}\right)$.

Proof. For all $k \in \mathbb{Z}_{+}$, we clearly have $T_{0} \leq T_{k}$. Also, $C_{c}^{\infty}(M) \subset Q\left(T_{k}\right)$ for all $k \in \mathbb{Z}_{+}$. By definition of $T_{0}$ it follows that $C_{c}^{\infty}(M)$ is dense in $Q\left(T_{0}\right)$ (in the norm of $\left.Q\left(T_{0}\right)\right)$.

Clearly, for all $w \in C_{c}^{\infty}(M)$,

$$
\left(w, T_{k} w\right) \rightarrow\left(w, T_{0} w\right) \quad \text { as } k \rightarrow \infty .
$$

We now apply Theorem 7.9 in 3 to conclude the proof of (i).

Property (ii) follows immediately since $T_{+} \geq T_{0}$.

2.17. Proof of Theorem 2.2, We will adopt the arguments from the proof of Theorem 1 in [2].

By the proof of Lemma 2.16 it follows that

$$
Q\left(T_{+}\right) \subset Q\left(T_{k}\right) \subset Q\left(T_{0}\right)
$$

and

$$
\|\cdot\|_{t_{0}} \leq\|\cdot\|_{t_{k}} \leq\|\cdot\|_{t_{+}}
$$

where $\|\cdot\|_{t_{0}},\|\cdot\|_{t_{k}}$ and $\|\cdot\|_{t_{+}}$are the norms associated to $t_{0}, t_{k}$ and $t_{+}$, respectively; cf. (1.2).

In fact, $Q\left(T_{k}\right)=Q\left(T_{+}\right)$since the norms $\|\cdot\|_{t_{k}}$ and $\|\cdot\|_{t_{+}}$are equivalent, because $V_{0}^{+}-V_{0}^{k}$ and $V_{0}^{+}$differ by a bounded function.

By Proposition 2.4, $T_{+}$has a positive form core, i.e. for every $u \in Q\left(T_{+}\right)^{+}$there exists a sequence $\phi_{j} \in C_{c}^{\infty}(M)^{+}$such that $\left\|\phi_{j}-u\right\|_{t_{+}} \rightarrow 0$ as $j \rightarrow \infty$. By (2.16) it follows that

$$
\left\|\phi_{j}-u\right\|_{t_{0}} \rightarrow 0 \quad \text { as } j \rightarrow \infty
$$

To prove the theorem, it remains to show that for every $w \in Q\left(T_{0}\right)^{+}$, there exists a sequence $w_{j} \in Q\left(T_{+}\right)^{+}$such that

$$
\left\|w_{j}-w\right\|_{t_{0}} \rightarrow 0 \quad \text { as } j \rightarrow \infty .
$$

Let $w \in Q\left(T_{0}\right)^{+}$. For every $k, l \in \mathbb{Z}_{+}$define

$$
w_{l}:=\left(\frac{1}{l} T_{0}+1\right)^{-1} w
$$

and

$$
w_{l}^{k}:=\left(\frac{1}{l} T_{k}+1\right)^{-1} w .
$$

This makes sense since $0 \leq T_{0} \leq T_{k}$ are self-adjoint operators.

By Lemma 2.12, the operator $\left(T_{k}+1\right)^{-1}$ is positivity preserving. Hence $w_{l}^{k} \in$ $\operatorname{Dom}\left(T_{k}\right)^{+} \subset Q\left(T_{k}\right)^{+}=Q\left(T_{+}\right)^{+}$.

Since the operators $\left(T_{0}+1\right)^{1 / 2}$ and $\left(T_{0} / l+1\right)^{-1}$ commute, we have

$$
\left\|w_{l}-w\right\|_{t_{0}}=\left\|\left(\left(\frac{1}{l} T_{0}+1\right)^{-1}-1\right)\left(T_{0}+1\right)^{1 / 2} w\right\|,
$$

where $\|\cdot\|$ denotes $L^{2}(M)$ norm. 
Clearly,

$$
\left(\frac{1}{l} T_{0}+1\right)^{-1} \rightarrow 1 \quad \text { strongly as } l \rightarrow \infty .
$$

This and (2.18) show that

$$
\left\|w_{l}-w\right\|_{t_{0}} \rightarrow 0 \quad \text { as } l \rightarrow \infty .
$$

Fix $l \in \mathbb{Z}_{+}$. For each $k \in \mathbb{Z}_{+}$, let $t_{0}+l$ and $t_{k}+l$ denote the quadratic forms corresponding to (positive self-adjoint) operators $T_{0}+l$ and $T_{k}+l$, respectively. Let $\|\cdot\|_{t_{0}+l}$ and $\|\cdot\|_{t_{k}+l}$ denote the norms in $Q\left(T_{0}+l\right)$ and $Q\left(T_{k}+l\right)$, respectively; cf. (1.2). The corresponding inner products will be denoted by $(\cdot, \cdot)_{t_{0}+l}$ and $(\cdot, \cdot)_{t_{k}+l}$.

Using (2.15), (2.16) and the Cauchy-Schwarz inequality we have for all $w \in$ $Q\left(T_{0}\right)^{+}$

$$
\begin{gathered}
=\left\|\left(T_{k}+l\right)^{-1} w\right\|_{t_{0}+l}^{2}+\left\|\left(T_{0}+l\right)^{-1} w\right\|_{t_{0}+l}^{2}-2\left(\left(T_{k}+l\right)^{-1} w,\left(T_{0}+l\right)^{-1} w\right)_{t_{0}+l} \\
\leq\left\|\left(T_{k}+l\right)^{-1} w\right\|_{t_{k}+l}^{2}+\left\|\left(T_{0}+l\right)^{-1} w\right\|_{t_{0}+l}^{2}-2\left(\left(T_{k}+l\right)^{-1} w,\left(T_{0}+l\right)^{-1} w\right)_{t_{0}+l} \\
\quad=\left(\left(T_{0}+l\right)^{-1} w, w\right)-\left(\left(T_{k}+l\right)^{-1} w, w\right) \\
+(1-l)\left[\left\|\left(T_{k}+l\right)^{-1} w\right\|^{2}+\left\|\left(T_{0}+l\right)^{-1} w\right\|^{2}-2\left(\left(T_{k}+l\right)^{-1} w,\left(T_{0}+l\right)^{-1} w\right)\right] \\
\leq\left(\left(T_{0}+l\right)^{-1} w, w\right)-\left(\left(T_{k}+l\right)^{-1} w, w\right) \leq\left\|\left(T_{0}+l\right)^{-1} w-\left(T_{k}+l\right)^{-1} w\right\|\|w\|,
\end{gathered}
$$

where $(\cdot, \cdot)$ is the inner product in $L^{2}(M)$ and $\|\cdot\|$ is the norm in $L^{2}(M)$.

By Lemma 2.16, it follows that for fixed $l \in \mathbb{Z}_{+}, T_{k}+l \rightarrow T_{0}+l$ in the strong resolvent sense as $k \rightarrow \infty$.

Clearly, for any positive self-adjoint operator $A,(A / l+1)^{-1}=l(A+l)^{-1}$. Therefore by (2.20), for a fixed $l \in \mathbb{Z}_{+}$,

$$
\left\|w_{l}^{k}-w_{l}\right\|_{t_{0}+l} \rightarrow 0 \quad \text { as } k \rightarrow \infty .
$$

This is equivalent to

$$
\left\|w_{l}^{k}-w_{l}\right\|_{t_{0}} \rightarrow 0 \quad \text { as } k \rightarrow \infty .
$$

Since $w_{l}^{k} \in Q\left(T_{+}\right)^{+}$, we can use (2.19) and (2.21) to choose a subsequence $\left\{w_{j}\right\}$ from $\left\{w_{l}^{k}\right\}$ so that (2.17) holds.

This concludes the proof of the theorem.

\section{Proof of Theorem 1.5}

We essentially follow the proof of Theorem 2 in [2]; however, we need to use Kato inequality (2.10) for operators on manifolds.

Without loss of generality, we may and we will assume that $\Delta_{M}+V_{0} \geq 0$ and $V_{1} \geq 0$.

Let us denote $T_{q}:=T_{0} \tilde{+} V_{1}$ and let $t_{\min }$ and $t_{q}$ be as in Sections 1.4 and 1.3 Since $t_{\min }$ and $t_{q}$ coincide on $C_{c}^{\infty}(M)$, it is sufficient to show that $C_{c}^{\infty}(M)$ is dense in the Hilbert space $Q\left(T_{q}\right)=Q\left(T_{0}\right) \cap Q\left(V_{1}\right)$ with the inner product

$$
(\cdot, \cdot)_{t_{q}}:=t_{q}(\cdot, \cdot)+(\cdot, \cdot)_{L^{2}(M)},
$$

where $t_{q}(\cdot, \cdot)$ is the sesquilinear form obtained by polarization of $t_{q}$.

Let $v \in Q\left(T_{q}\right)$ be orthogonal to $C_{c}^{\infty}(M)$ in $(\cdot, \cdot)_{t_{q}}$. This means that for all $w \in C_{c}^{\infty}(M)$,

$$
\left(\left(\Delta_{M}+V_{0}+V_{1}\right) v, w\right)_{L^{2}(M)}+(v, w)_{L^{2}(M)}=0 .
$$


This leads to the following distributional equality:

$$
\Delta_{M} v=-\left(V_{0}+V_{1}+1\right) v .
$$

Since $V_{1} \in L_{\mathrm{loc}}^{1}(M)$ and $v \in Q\left(V_{1}\right)$, we have

$$
2\left|V_{1} v\right|=2\left|V_{1}\right||v| \leq\left|V_{1}\right|+\left|V_{1}\right||v|^{2}
$$

which immediately gives $V_{1} v \in L_{\mathrm{loc}}^{1}(M)$.

Since $V_{0} \in L_{\text {loc }}^{2}(M)$, it follows that $V_{0} v \in L_{\text {loc }}^{1}(M)$. From (3.1) we obtain $\Delta_{M} v \in L_{\mathrm{loc}}^{1}(M)$.

Using Kato inequality (2.10) in case $\nabla=d$ and (3.1), we get

$$
\Delta_{M}|v| \leq \operatorname{Re}\left(\operatorname{sign} \bar{v} \Delta_{M} v\right)=-V_{0}|v|-V_{1}|v|-|v| \leq-\left(V_{0}+1\right)|v| .
$$

The last inequality in (3.2) holds since $V_{1} \geq 0$.

From (3.2), we obtain the following distributional inequality:

$$
\left(\Delta_{M}+V_{0}+1\right)|v| \leq 0 .
$$

Let $T_{0}$ be as in the hypothesis, and let $t_{0}$ denote the closed quadratic form associated to $T_{0}$.

Using (3.3), we get

$$
\left(\left(T_{0}+1\right) w,|v|\right)_{L^{2}(M)} \leq 0 \quad \text { for all } w \in C_{c}^{\infty}(M)^{+} .
$$

Since $v \in Q\left(T_{0}\right)$, Corollary 2.14 gives $|v| \in Q\left(T_{0}\right)$. Therefore, we can write (3.4) as

$$
(w,|v|)_{t_{0}} \leq 0 \quad \text { for all } w \in C_{c}^{\infty}(M)^{+},
$$

where $(\cdot, \cdot)_{t_{0}}=t_{0}(\cdot, \cdot)+(\cdot, \cdot)_{L^{2}(M)}$ denotes the inner product in $Q\left(T_{0}\right)$.

Let $f:=\left(T_{0}+1\right)^{-1}|v|$. By Proposition $2.13\left(T_{0}+1\right)^{-1}$ is positivity preserving, so $f \in \operatorname{Dom}\left(T_{0}\right)^{+} \subset Q\left(T_{0}\right)^{+}$.

By Theorem 2.2 $T_{0}$ has a positive form core. Therefore, there exists a sequence $f_{k} \in C_{c}^{\infty}(M)^{+}$such that

$$
\lim _{k \rightarrow \infty}\left(f_{k},|v|\right)_{t_{0}}=(f,|v|)_{t_{0}}=\left(\left(T_{0}+1\right)^{-1}|v|,|v|\right)_{t_{0}}=\|v\|^{2},
$$

where $v$ and $(\cdot, \cdot)_{t_{0}}$ are as in (3.5), and $\|\cdot\|$ is the norm in $L^{2}(M)$.

From (3.5) and (3.6) we obtain $\|v\|^{2} \leq 0$, i.e. $v=0$.

This shows that $C_{c}^{\infty}(M)$ is dense in $Q\left(T_{q}\right)$, and the theorem is proven.

\section{REFERENCES}

[1] M. Braverman, O. Milatovic, M. A. Shubin, Essential self-adjointness of Schrödinger type operators on manifolds, Russian Math. Surveys, 57 (4) (2002), 641-692.

[2] H. L. Cycon, On the form sum and the Friedrichs extension of Schrödinger operators with singular potentials, J. Operator Theory, 6 (1981), 75-86. MR 82k:47068

[3] W. G. Faris, Self-adjoint Operators, Lecture Notes in Mathematics No. 433, Springer-Verlag, Berlin e.a., 1975. MR 57:7207

[4] D. Gilbarg, N. S. Trudinger, Elliptic partial differential equations of second order, Springer, New York, 1977. MR 57:13109

[5] H.-W. Goelden, On non-degeneracy of the ground state of Schrödinger operators, Math. Z., 155 (1977), 239-247. MR 58:29426

[6] H. Hess, R. Schrader, D.A. Uhlenbrock, Domination of semigroups and generalization of Kato's inequality, Duke Math. J., 44 (1977), 893-904. MR 56:16446

[7] H. Hess, R. Schrader, D. A. Uhlenbrock, Kato's inequality and the spectral distribution of Laplacians on compact Riemannian manifold, J. Differential Geom., 15 (1980), 27-37. MR 82g:58090

[8] T. Kato, Perturbation theory for linear operators, Springer-Verlag, New York, 1980. reprint MR 96a:47025 
[9] T. Kato, A second look at the essential selfadjointness of the Schrödinger operators, Physical reality and mathematical description, Reidel, Dordrecht, 1974, 193-201. MR 57:16958

[10] M. Reed, B. Simon, Methods of Modern Mathematical Physics I, II: Functional analysis. Fourier analysis, self-adjointness, Academic Press, New York e.a., 1975. MR 58:12429a MR 58:12429b

[11] B. Simon, An abstract Kato's inequality for generators of positivity preserving semigroups, Indiana Univ. Mat. J., 26 (1977), 1067-1073. MR 57:1194

[12] B. Simon, Kato's inequality and the comparison of semigroups, J. Funct. Anal., 32 (1979), 97-101. MR 80e:47036

[13] B. Simon, Maximal and minimal Schrödinger forms, J. Operator Theory, 1 (1979), 37-47. MR 81m:35104

[14] M. Taylor, Partial Differential Equations II: Qualitative Studies of Linear Equations, Springer-Verlag, New York e.a., 1996. MR 98b:35003

Department of Mathematics, Northeastern University, Boston, Massachusetts 02115 E-mail address: ogmilato@lynx.neu.edu

Current address: Department of Mathematics, Fitchburg State College, Fitchburg, Massachusetts 01420

E-mail address: omilatovic@fsc.edu 